\title{
CARACTERIZAÇÃO ELEMENTAR DA CASCA DE ARROZ E SUAS CINZAS POR MIP OES APÓS DECOMPOSIÇÃO ÁCIDA COM SISTEMA DE REFLUXO
}

\author{
Emanoelli R. Lopesa, Aline L. Medinaa ${ }^{a}$ Anderson S. Ribeiro ${ }^{a}$, João N. Brandalise ${ }^{\mathrm{b}}$ e Adriane M. Nunes ${ }^{\mathrm{a}, *}$ \\ ${ }^{a}$ Centro de Ciências Químicas, Farmacêuticas e de Alimentos, Universidade Federal de Pelotas, Campus Capão do Leão, 96160-000 \\ Capão do Leão - RS, Brasil \\ 'Instituto de Biologia, Departamento de Ecologia, Zoologia e Genética, Universidade Federal de Pelotas, Campus Capão do Leão, \\ 96160-000 Capão do Leão - RS, Brasil
}

Recebido em 15/04/2017, aceito em 24/07/2017, publicado na web em 12/09/2017

\begin{abstract}
ELEMENTARY CHARACTERIZATION OF RICE HUSK AND THEIR ASHES BY MIP OES AFTER ACID DECOMPOSITION WITH REFLUX SYSTEM. This paper describes the optimization of a sample preparation method using response surface methodology for $\mathrm{Al}, \mathrm{Cd}, \mathrm{Cu}, \mathrm{Fe}, \mathrm{Mg}, \mathrm{Mn}, \mathrm{Mo}, \mathrm{Na}, \mathrm{Ni}$ and Ti determinations in samples of rice husk and rice husk ash by MIP OES. Sample preparation was performed using a reflux system adapted to the digestion tubes. This system allowed the decomposition of the samples without the necessity of replacement of the acids during this step without any loss of volatile elements. The decompositions were performed with $3.6 \mathrm{~mL}$ of $\mathrm{HNO} 3$ and $1.4 \mathrm{~mL}$ of $\mathrm{H}_{2} \mathrm{SO}_{4}$ for $205 \mathrm{~min}$ in a digester block at $225{ }^{\circ} \mathrm{C}$. The detection limit for determination of respective analytes in both samples were satisfactory. The RSDs for the analytes were less than $10 \%$. Moreover, the accuracy of the method can be verified by recovery tests, whose values ranged from 82 to $115 \%$ for husk samples and 82 to $120 \%$ for ashes. The proposed method was presented as a simple, safe and low cost procedure being suitable for sample preparation and subsequent determination of metals by MIP OES.
\end{abstract}

Keywords: rice husk and rice husk ashes, reflux system, metals, MIP OES.

\section{INTRODUÇÃO}

De acordo com o Ministério da Agricultura Pecuária e Abastecimento (MAPA) o arroz está entre os cereais mais consumidos no mundo, sendo o Brasil o nono maior produtor mundial de arroz, com mais de $50 \%$ da sua produção localizada no estado do Rio Grande do Sul. ${ }^{1}$

$\mathrm{Na}$ indústria do arroz temos, como subproduto mais volumoso, a casca do grão, que corresponde a cerca de $20 \%$ do peso do grão de arroz. ${ }^{2}$ Por esta razão, é necessário encontrar formas de reutilização para este subproduto. Por apresentar um alto poder calorífico, cerca de $16,3 \mathrm{MJ} / \mathrm{Kg}$, a queima da casca de arroz para a geração de energia se apresenta como uma alternativa atrativa do ponto de vista econômico e viável em termos tecnológicos, além de apresentar baixos impactos ambientais, visto que todo $\mathrm{o} \mathrm{CO}_{2}$ produzido na queima volta para o ciclo de carbono da biosfera terrestre. ${ }^{3}$

No entanto, através da queima deste subproduto, uma quantidade significativa de cinzas é gerada como resíduo. Tendo em vista que grande parte dessas cinzas apresentam características pozolânicas, estas podem ser utilizadas na indústria cimenteira. ${ }^{4}$ Outra forma de aplicação das cinzas da casca de arroz é na agricultura, por conta dos minerais presentes na sua composição. Por ser um material rico em sílica as cinzas também podem ser utilizadas na fabricação de cerâmica e na indústria automotiva. ${ }^{5}$

A casca de arroz apresenta um teor de cinzas de aproximadamente $11 \%$, sendo que estas cinzas contém em torno de $80-90 \%$ de $\mathrm{SiO}_{2}, 5 \%$ de $\mathrm{K}_{2} \mathrm{O}, 4 \%$ de $\mathrm{P}_{2} \mathrm{O}_{5}$ e $1-2 \%$ de $\mathrm{CaO}$ e quantidades menores de $\mathrm{Fe}$, $\mathrm{Mg}$ e Na. Contudo, em sua composição os principais componentes orgânicos são a celulose (43,5\%), a hemicelulose (22\%) e a lignina $(17,2 \%) .{ }^{6}$ Pequenas diferenças na composição química da casca de arroz são esperadas e estas são dependentes da safra, do clima, bem como das condições do solo e localização geográfica. ${ }^{7}$

*e-mail: adriane.mn@hotmail.com
Por se tratar de uma mistura complexa de alto teor orgânico, associado a componentes inorgânicos como silicatos, óxidos, entre outros, a casca de arroz pode apresentar em sua composição a presença de elementos tóxicos. Sendo assim, o conhecimento do conteúdo desta matriz, bem como de seus subprodutos, pode fornecer informações preciosas para avaliar o risco ambiental embutido, devido ao crescente aumento do uso da casca de arroz como biomassa, aliado à necessidade de obtenção de uma cinza de melhor qualidade para que esta possa ser utilizada em diversos setores industriais.

O procedimento de decomposição comumente utilizado para matrizes com essas características, é a decomposição ácida por via úmida. Desta forma, para uma completa solubilização das amostras o uso de misturas de ácidos minerais a quente, normalmente, se faz necessário. ${ }^{8}$

Dentro deste contexto, o uso de sistemas de refluxo para o preparo de amostras tem se destacado como uma alternativa promissora aos sistemas convencionais de decomposição ácida. Como vantagens pode-se citar o processo de condensação dos ácidos, o que evita a necessidade de reposição destes reagentes durante a etapa de decomposição. O refluxo da solução acaba também minimizando os riscos de contaminação, bem como a perda de analito por volatilização. ${ }^{9-11}$

Em busca de otimizar os processos e as metodologias analíticas para obter resultados exatos e precisos, e ainda possibilitar uma diminuição dos custos da análise e o tempo empregado, as técnicas sistemáticas de planejamento estatístico de experimentos vêm sendo cada vez mais utilizadas nos estudos experimentais. A metodologia de delineamento composto central rotacional (DCCR) permite, com um número reduzido de ensaios, avaliar as inter-relações entre as variáveis e as respostas obtidas, através da geração de gráficos de superfície de resposta para análise dos resultados. ${ }^{12}$

Frente a esta problemática, e por se tratar de matrizes complexas, fica evidente a necessidade do desenvolvimento e da validação de métodos analíticos voltados principalmente ao estudo da etapa de preparo da amostra para posterior determinação elementar. Desta 
forma, o presente trabalho tem como objetivo principal o desenvolvimento de um método analítico de decomposição ácida sob refluxo para posterior determinação de metais em amostras de casca e cinzas da casca de arroz por espectrometria de emissão atômica com plasma induzido por micro-ondas (MIP OES). Para a otimização das melhores condições de trabalho o experimento foi realizado a partir de um planejamento estatístico DCCR.

\section{PARTE EXPERIMENTAL}

\section{Equipamentos e parâmetros instrumentais}

Para as análises foi utilizado um espectrômetro de emissão atômica com plasma induzido por micro-ondas (MIP OES) modelo Agilent 4200 (Agilent Technologies, Melbourne, Austrália) equipado com uma câmara de pulverização ciclônica de vidro de dupla passagem (Agilent Technologies, Melbourne, Austrália). O nitrogênio utilizado é extraído do ar atmosférico por meio de um gerador de nitrogênio (4107 Nitrogen Generator - Agilent Technologies, Melbourne, Austrália). O alinhamento da tocha e a seleção da melhor linha espectral foram realizadas utilizando uma solução de calibração de $\mathrm{HNO}_{3} 5 \%$ e otimizados automaticamente pelo instrumento com o MP Expert Software (versão 1.5.1.0, Agilent Technologies, Melbourne, Austrália). Os elementos quantificados e os parâmetros instrumentais utilizados estão presentes na Tabela 1.

As amostras foram trituradas em um multiprocessador de alimentos convencional e peneiradas em uma peneira de malha 300 $\mu \mathrm{m}$ (Bronzinox, São Paulo, Brasil). Em seguida, as amostras foram pesadas utilizando uma balança analítica com resolução de $0,1 \mathrm{mg}$ e tara máxima de $210 \mathrm{~g}$ (Modelo AR 2140, Ohaus Adventurer, EUA). Para a etapa de decomposição ácida das amostras foi utilizado um bloco digestor convencional (modelo MA-4025, Marconi, Brasil), onde foi adaptado a cada um dos tubos digestores um sistema de refluxo.

Este sistema consiste na inserção de um tubo pequeno de vidro, chamado de "dedo frio", o qual é adaptado ao tubo de digestão do bloco digestor convencional. A água é circulada de forma contínua dentro deste tubo, onde a temperatura é mantida a $15^{\circ} \mathrm{C}$, sendo esta controlada através de um banho termostatizado modelo Q-214M2 (Quimis, São Paulo, Brasil). Desta forma, ocorre o resfriamento da parte superior do tubo digestor, o que proporciona a recirculação dos ácidos dentro do tubo maior. O sistema é semifechado com uma tampa de Politetrafluoretileno (PTFE), a qual contém uma ranhura lateral que possibilita o alivio da pressão no interior do tubo, diminuindo ou anulando qualquer risco de explosão, tornando-se um método mais seguro ao analista.

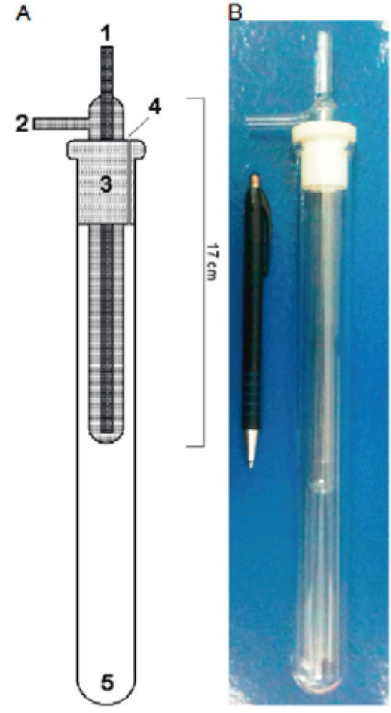

Figura 1. A) Diagrama esquemático do sistema de refluxo acoplado ao tubo digestor de vidro. 1: entrada água; 2: saída de água; 3: Tampa de Teflon ${ }^{\circledR}$;: saída para gás; 5: tubo de reação. B) Imagem real do sistema de dedo frio. (adaptado de Orestes et al. 2013) ${ }^{9}$

\section{Reagentes e soluções}

Todos os reagentes utilizados foram de grau analítico: ácido nítrico $65 \%(\mathrm{~m} / \mathrm{m})$ (Synth, São Paulo, Brasil) foi purificado por destilação em um sistema de sub-ebulição de quartzo MA-075 (Marconi, Piracicaba, Brasil), ácido sulfúrico $98 \%(\mathrm{~m} / \mathrm{m})$ (Vetec, Rio de Janeiro, Brasil). Os materiais de vidro foram descontaminados em um banho de $10 \%$ (v/v) de $\mathrm{HNO}_{3}$ por um período de 48 horas, e após, foram enxaguados com água deionizada, antes do uso. A água utilizada no preparo das amostras e das soluções foi obtida por um sistema de purificação com resina de troca iônica. Para a calibração do instrumento foi utilizado uma solução padrão multielementar para ICP (Multielement Standard Solution6 for ICP - Sigma Aldrish, Buchs, Alemanha).

\section{Preparo das amostras}

As amostras de casca e cinza de casca de arroz foram cedidas por três empresas de beneficiamento de arroz, localizadas na região sul do estado do Rio Grande do Sul. Por estas empresas foram disponibilizadas cinco amostras de casca e cinco amostras de cinzas, sendo estas coletadas em dias diferentes durante uma semana, totalizando quinze amostras de casca e quinze amostras de cinzas.

Tabela 1. Condições operacionais de trabalho utilizadas na determinação de $\mathrm{Al}, \mathrm{Cd}, \mathrm{Cu}, \mathrm{Fe}, \mathrm{Mg}, \mathrm{Mn}, \mathrm{Mo}, \mathrm{Na}$, Ni e Ti por MIP OES.

\begin{tabular}{|c|c|c|c|c|c|c|c|c|c|c|c|}
\hline \multirow{2}{*}{ Parâmetros } & \multicolumn{11}{|c|}{ Elementos } \\
\hline & $\mathrm{Al}$ & $\mathrm{Cd}$ & $\mathrm{Cu}$ & $\mathrm{Fe}$ & $\mathrm{Mg}$ & $\mathrm{Mn}$ & Mo & $\mathrm{Na}$ & $\mathrm{Ni}$ & Co & $\mathrm{Ti}$ \\
\hline Comprimento de onda (nm) & 396,152 & 228,802 & 324,754 & 371,993 & 282,213 & 403,076 & 379,825 & 588,995 & 352,454 & 340,512 & 334,941 \\
\hline Posição de observação & -10 & 10 & 0 & 0 & 10 & 0 & 0 & 0 & 0 & 0 & 10 \\
\hline Fluxo do nebulizador (L/min) & 1 & 0,55 & 0,6 & 0,75 & 0,7 & 0,85 & 0,75 & 1 & 0,6 & 0,65 & 0,6 \\
\hline Tempo de leitura (s) & & & & & & 3 & & & & & \\
\hline Numero de replicatas & & & & & & 3 & & & & & \\
\hline Tempo de captação da amostra (s) & & & & & & 15 & & & & & \\
\hline Tempo de estabilização (s) & & & & & & 15 & & & & & \\
\hline Velocidade da bomba (rpm) & & & & & & 15 & & & & & \\
\hline Acionamento rápido da bomba & & & & & & Ligado & & & & & \\
\hline Corretor de fundo & & & & & & Auto & & & & & \\
\hline Introdução da amostra & & & & & & Manual & & & & & \\
\hline
\end{tabular}


Todas as amostras analisadas foram submetidas a uma etapa de moagem em um multiprocessador de alimentos, até ficarem na forma de pó. Após, $250 \mathrm{mg}$ de cada amostra de casca de arroz foram pesadas, homogeneizadas e armazenadas no mesmo recipiente, formando uma amostra composta, a qual foi utilizada para o desenvolvimento e validação do método proposto. No entanto, para a validação das amostras de cinzas utilizou-se uma amostra cedida pela segunda empresa que foi coletada anteriormente as 15 amostras utilizadas para as analises.

Posteriormente, foi realizado um planejamento estatístico através do software Statistica 7.0 (StatSoft, Inc., Tulsa, OK, EUA) para compreender a influência de cada parâmetro da etapa de preparo de amostras sobre a variação do sinal analítico de cada elemento. Este planejamento possibilita uma melhor otimização das condições de trabalho, de forma que seja possível estabelecer uma condição compromisso através das variáveis (variáveis independentes) em estudo: porcentagem de ácido nítrico (\% Ac) em relação a quantidade de ácido sulfúrico em um volume total de $5 \mathrm{~mL}$, tempo de decomposição no bloco digestor (t) e temperatura do bloco digestor (T). Após definir as variáveis em estudo, um delineamento composto central rotacional (DCCR), $2^{3}$ com 3 pontos centrais e 6 pontos axiais foi efetuado gerando um total de 17 ensaios, os quais foram realizados de acordo com as análises arranjadas pelo software Statistica 7.0 de forma aleatória e em triplicata. As respostas escolhidas (variáveis dependentes) correspondem aos sinais analíticos obtidos para os elementos $\mathrm{Fe}, \mathrm{Mg}$, $\mathrm{Mn}$ e $\mathrm{Na}$

\section{Método proposto com o sistema de refluxo}

Foi fixada uma condição de trabalho para as amostras de casca de arroz, satisfatória para todos os elementos em estudo, com base na análise dos gráficos de superfície de resposta obtidos para cada elemento. Essa condição foi aplicada também para as amostras de cinzas de casca de arroz, visto que são amostras menos complexas que as amostras de casca, pois grande parte da carga orgânica é eliminada durante a queima da casca.

Sendo assim, foram pesadas aproximadamente $250 \mathrm{mg}$ de amostras diretamente nos tubos digestores, em seguida foi adicionado 3,6 mL de $\mathrm{HNO}_{3}$ e $1,4 \mathrm{~mL}$ de $\mathrm{H}_{2} \mathrm{SO}_{4}$, a seguir, os tubos foram levados ao bloco digestor com temperatura de $225^{\circ} \mathrm{C}$, já com o sistema de refluxo acoplado aos tubos digestores, utilizando uma recirculação interna de água a qual foi mantida a $15{ }^{\circ} \mathrm{C}$, por 3 horas e $25 \mathrm{~min}$. Ao término deste período, as soluções resultantes nos tubos digestores foram transferidas de forma quantitativa para frascos de PP (polipropileno), previamente descontaminados, e estes aferidos a 50 $\mathrm{mL}$ com água deionizada. Uma diluição foi realizada a partir dessa solução, com intuito de adequar a acidez da amostra de modo a não causar danos ao equipamento, onde $5 \mathrm{~mL}$ foram transferidos para outro frasco de PP e aferidos a $10 \mathrm{~mL}$ de água deionizada. Todas as análises foram realizadas em replicata, para verificar a precisão do método proposto, e testes de adição e recuperação de analito, com as duas amostras em estudo, foram feitos com o propósito de verificar a exatidão do método. Por fim, foi feita a aplicação do método em 15 amostras de casca de arroz e 15 amostras de cinzas de casca de arroz.

\section{RESULTADOS E DISCUSSÕES}

\section{Otimização do método proposto}

Para uma melhor otimização das condições de trabalho do método proposto de digestão ácida sob refluxo um DCCR foi realizado. Para estes estudos, conforme citado anteriormente, foi utilizada uma amostra composta de casca de arroz. Os níveis utilizados neste delineamento foram estabelecidos a partir de testes experimentais feitos previamente e com base na literatura. O DCCR conta com as seguintes variáveis independentes: porcentagem de $\mathrm{HNO}_{3}$ (em relação ao ácido sulfúrico), tempo de permanência dos tubos no bloco digestor e temperatura do bloco digestor, representados na Tabela 2. Os valores de porcentagem de 40, 48, 60, 72 e 80\% descritos na referida Tabela, referem-se respectivamente aos volumes de $2 \mathrm{~mL}$ de $\mathrm{HNO}_{3}$ e $3 \mathrm{~mL} \mathrm{H}_{2} \mathrm{SO}_{4}, 2,4 \mathrm{~mL}$ de $\mathrm{HNO}_{3}$ e $2,6 \mathrm{~mL} \mathrm{H}_{2} \mathrm{SO}_{4}, 3 \mathrm{~mL}$ de $\mathrm{HNO}_{3}$ e $2 \mathrm{~mL} \mathrm{H}_{2} \mathrm{SO}_{4}, 3,6 \mathrm{~mL}$ de $\mathrm{HNO}_{3}$ e $1,4 \mathrm{~mL} \mathrm{H}_{2} \mathrm{SO}_{4}$ e por fim

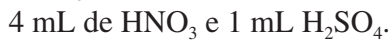

Tabela 2. Variáveis e valores utilizados no DCCR

\begin{tabular}{lccccc}
\hline \multirow{2}{*}{ Variáveis independentes } & \multicolumn{5}{c}{ Nível } \\
\cline { 2 - 6 } & $-1,68$ & -1 & 0 & 1 & 1,68 \\
\hline Porcentagem de $\mathrm{HNO}_{3}(\%)$ & 40 & 48 & 60 & 72 & 80 \\
Tempo (min) & 60 & 95 & 150 & 205 & 240 \\
Temperatura $\left(\mathrm{C}^{\circ}\right)$ & 150 & 180 & 225 & 270 & 300
\end{tabular}

Através deste estudo foi gerado pelo software uma matriz DCCR com 17 ensaios, onde as variáveis estudadas são combinadas entre si como mostra a Tabela 3. Também estão representados, os resultados de emissão de sinal analítico obtidos para os elementos estatisticamente significativos, os quais foram analisados por MIP OES nas amostras de casca de arroz, após a etapa experimental de decomposição ácida.

Após análise das amostras por MIP OES e obtenção do sinal analítico dos elementos $\mathrm{Al}, \mathrm{Cd}, \mathrm{Cu}, \mathrm{Fe}, \mathrm{Mg}, \mathrm{Mn}, \mathrm{Mo}, \mathrm{Na}$, Ni e Ti, foi possível observar que somente os elementos $\mathrm{Fe}, \mathrm{Mg}$, Mo e $\mathrm{Na}$ mostraram respostas estatisticamente significativas. Com base nos Diagramas de Pareto (Figura 2), pôde-se observar que o Mg apresentou somente a $\% \mathrm{HNO}_{3}$ como variável estatisticamente significativa, enquanto que o Mo apresentou todas as variáveis estatisticamente significativas. Já para o Na, somente o tempo foi significativo. Para o Fe, o tempo e a interação entre o tempo e a \% Ac foram significativos. Abaixo, estão descritas as equações 1, 2, 3 e 4 correspondentes, respectivamente, aos modelos referentes à determinação para Fe, Mg, Mo e Na.

$y=47294,12-2573,5 t+5732,62 \%$ Ac. $t$
$y=170023,8+14872,8 \%$ Ac
$y=1671,540-573,580 \% A c^{2}-423,433 t^{2}-319,393 T^{2}$
$y=47749,77-7560,83 t^{2}$

Foi possível calcular o valor de $\mathrm{F}$ a partir dos dados da tabela ANOVA (Tabela 4), além de outros parâmetros importantes estatisticamente. Para todos analitos os valores de F calculados foram maiores que os valores de F tabelado. Com (Fcal>Ftab) foi possível gerar os gráficos de superfície de resposta para cada elemento e analisar como cada variável estatisticamente significativa influenciou na resposta do sinal emitido pelo analito, o que possibilitou encontrar uma possível condição compromisso, considerando as melhores condições de analise encontradas para cada analito.

De acordo com a Figura 3, foi possível observar que o Fe em relação às variáveis estatisticamente significativas (\% Ac e tempo) apresentou os melhores resultados para os valores próximos as extremidades, ou seja, para os maiores ou menores valores de \% Ac e tempo, sendo possível observar melhores sinais de absorbância com uma variação diretamente proporcional entre as duas variáveis.

Observando o gráfico de superfície de resposta obtido para o $\mathrm{Mg}$ (Figura 4), é possível observar que os melhores resultados foram obtidos quando os maiores valores $\% \mathrm{HNO}_{3}$ foram utilizados. Para o Mo (Figura 5 e 6), observa-se que os melhores resultados, para as três variáveis estatisticamente significativas \% Ac, temperatura e tempo, tendem ao ponto central. Para o Na (Figura 7), os resultados 
Tabela 3. Matriz do DCCR para o preparo de amostras de casca de arroz com o sistema de refluxo

\begin{tabular}{|c|c|c|c|c|c|c|c|}
\hline \multirow{2}{*}{ Ensaios } & \multicolumn{3}{|c|}{ Variáveis Independetes } & \multicolumn{4}{|c|}{ Variáveis Dependentes } \\
\hline & $\% \mathrm{Ac}$ & $\mathrm{T}$ & $\mathrm{T}$ & $\mathrm{Fe}$ & $\mathrm{Mg}$ & Mo & $\mathrm{Na}$ \\
\hline 1 & -1 & -1 & -1 & 63533,67 & 157540,71 & 0 & 13411,68 \\
\hline 2 & 1 & -1 & -1 & 51028,13 & 210115,88 & 0 & 18184,92 \\
\hline 3 & -1 & 1 & -1 & 40317,30 & 151406,73 & 0 & 22695,34 \\
\hline 4 & 1 & 1 & -1 & 51748,74 & 200769,23 & 0 & 33483,26 \\
\hline 5 & -1 & -1 & 1 & 53621,76 & 195918,00 & 0 & 35790,59 \\
\hline 6 & 1 & -1 & 1 & 47166,56 & 174556,59 & 141,26 & 23992,70 \\
\hline 7 & -1 & 1 & 1 & 34082,07 & 145133,68 & 0 & 45891,09 \\
\hline 8 & 1 & 1 & 1 & 49550,86 & 186956,65 & 215,05 & 64426,86 \\
\hline 9 & $-1,68$ & 0 & 0 & 41101,89 & 122548,11 & 351,68 & 63114,02 \\
\hline 10 & 1,68 & 0 & 0 & 42987,44 & 170542,34 & 625,24 & 63029,64 \\
\hline 11 & 0 & $-1,68$ & 0 & 43756,79 & 152079,21 & 751,74 & 31644,76 \\
\hline 12 & 0 & 1,68 & 0 & 46435,62 & 158678,42 & 1074,54 & 43582,70 \\
\hline 13 & 0 & 0 & $-1,68$ & 52562,98 & 156310,78 & 1237,56 & 42776,06 \\
\hline 14 & 0 & 0 & 1,68 & 46184,90 & 132003,91 & 1177,26 & 44635,37 \\
\hline 15 & 0 & 0 & 0 & 49744,75 & 188208,05 & 1479,90 & 62032,08 \\
\hline 16 & 0 & 0 & 0 & 44908,50 & 158226,67 & 1689,53 & 49626,82 \\
\hline 17 & 0 & 0 & 0 & 45267,99 & 169537,80 & 1694,46 & 50171,10 \\
\hline
\end{tabular}
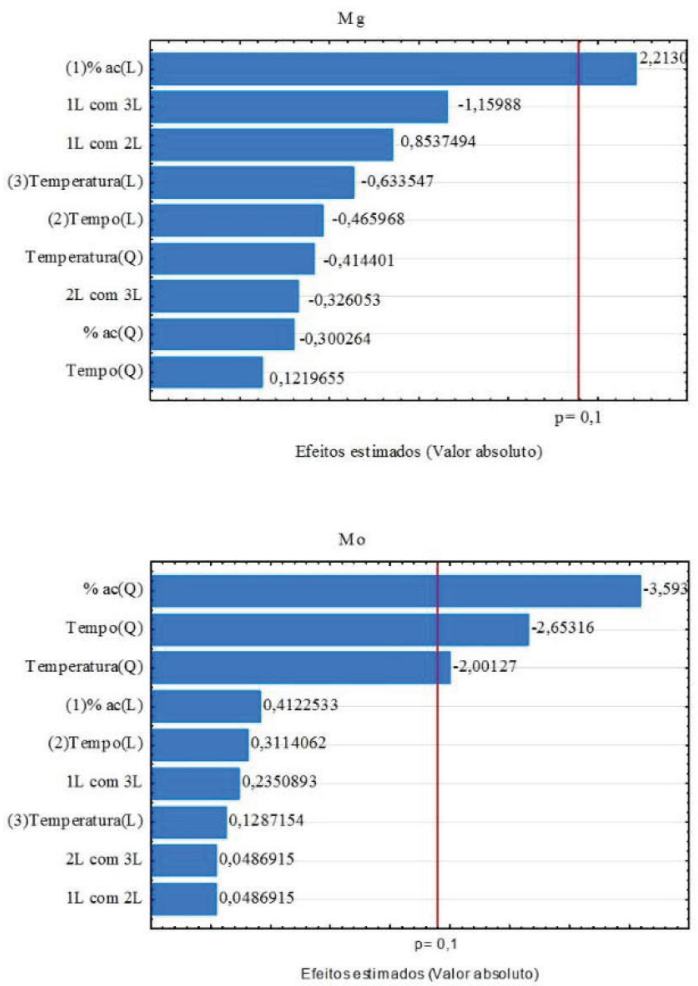

Figura 2. Gráficos de Pareto para $\mathrm{Fe}, \mathrm{Mg}$, $\mathrm{Mo}$ e $\mathrm{Na}$

foram semelhantes ao Mo para a variável tempo, apresentando como melhor resultado o ponto central.

Depois de observar os gráficos de superfície de resposta obtidos para os analitos em estudos, pôde-se chegar a uma condição compromisso de preparo de amostra, sendo esta satisfatória para realizar a determinação dos quatro elementos analisados. Dessa forma, a temperatura foi fixada em $225^{\circ} \mathrm{C}$, valor do ponto central, visto que esta variável só foi significativa para o Mo. O tempo foi definido em 205 min, em razão de que esta variável foi significativa para três dos quatro elementos analisados, sendo que para o $\mathrm{Na}$ e o Mo os melhores resultados foram para valores próximos ao ponto central e para o $\mathrm{Fe}$
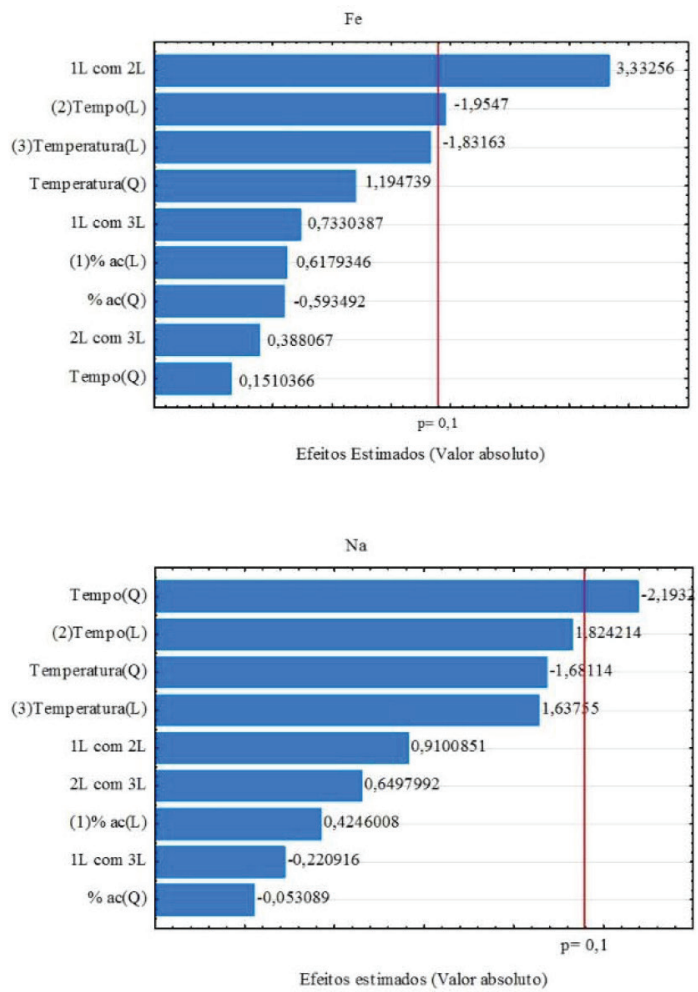

os melhores resultados foram nas extremidades, em razão disso foi escolhido o ponto intermediário entre a extremidade de maior valor e o ponto central, a extremidade de menor valor foi descartada pela possibilidade de um curto período de tempo de decomposição não ser suficiente para os demais elementos. $\mathrm{A} \% \mathrm{HNO}_{3}$ foi fixada em $72 \%$, que corresponde a $3,6 \mathrm{~mL}$ de $\mathrm{HNO}_{3}$ e $1,4 \mathrm{~mL}$ de $\mathrm{H}_{2} \mathrm{SO}_{4}$, pois os melhores resultados para o $\mathrm{Mg}$ e para o $\mathrm{Fe}$ foram obtidos na extremidade superior, no entanto o Mo apresentou os melhores resultados próximos ao ponto central, por essa razão foi a escolhida a condição intermediária, de $72 \%$.

Por conseguinte, a condição compromisso fixada para os 
Tabela 4. Análise de variância (ANOVA) para a determinação de Fe, Mg, Mo e Na em amostras de casca de arroz

\begin{tabular}{|c|c|c|c|c|c|c|}
\hline$\underline{\text { Respostas }}$ & Fonte de variação & Soma dos quadrados & Grau de liberdade & Quadrado médio & F cal & F tab \\
\hline & Regressão & 353351463,6 & 2 & 176675731,8 & 7,553136 & 2,726 \\
\hline \multirow[t]{3}{*}{$\mathrm{Fe}$} & Resíduos & 327474624,2 & 14 & 23391044,59 & & \\
\hline & Total & 680826088 & 16 & & & \\
\hline & Regressão & 3020896438 & 1 & 3020896438 & 7,274375 & 3,073 \\
\hline \multirow[t]{3}{*}{$\mathrm{Mg}$} & Resíduos & 6229187974 & 15 & 415279198,3 & & \\
\hline & Total & $9,250084 \mathrm{E}+09$ & 16 & & & \\
\hline & Regressão & 4616033,48 & 3 & 1538677,827 & 9,486182 & 2,56 \\
\hline \multirow[t]{3}{*}{ Mo } & Resíduos & 2108626,234 & 13 & 162202,018 & & \\
\hline & Total & 6724660 & 16 & & & \\
\hline & Regressão & 744808112,8 & 1 & 744808112,8 & 3,196112 & 3,073 \\
\hline \multirow[t]{2}{*}{$\mathrm{Na}$} & Resíduos & 3495534696 & 15 & 233035646,4 & & \\
\hline & Total & $4,240343 E+09$ & 16 & & & \\
\hline
\end{tabular}

$\mathrm{F}_{1,15}=3,073 ; \mathrm{F}_{3,13}=2,560 ; \mathrm{F}_{2,14}=2,726$.

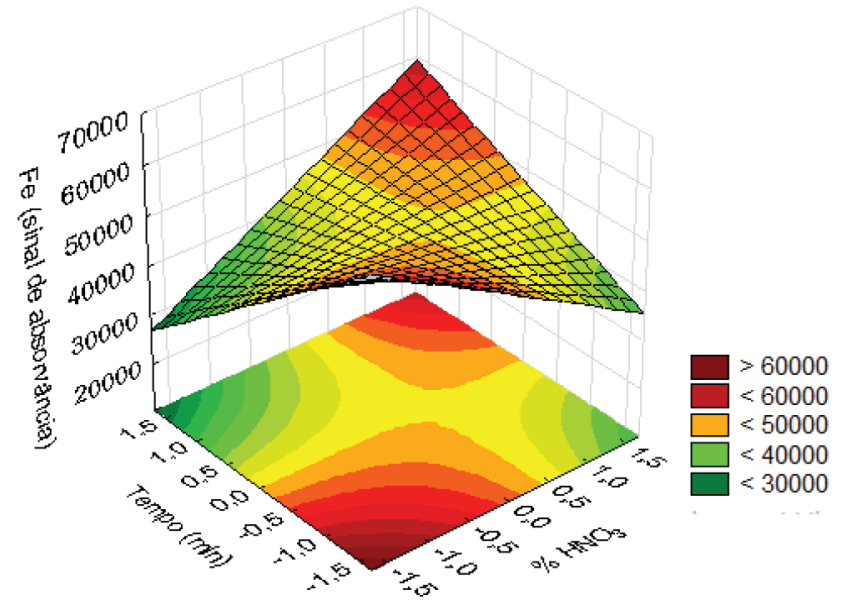

Figura 3. Gráficos de superfície de resposta em função do sinal analítico do $\mathrm{Fe}(\mathrm{tx} \% \mathrm{Ac})$

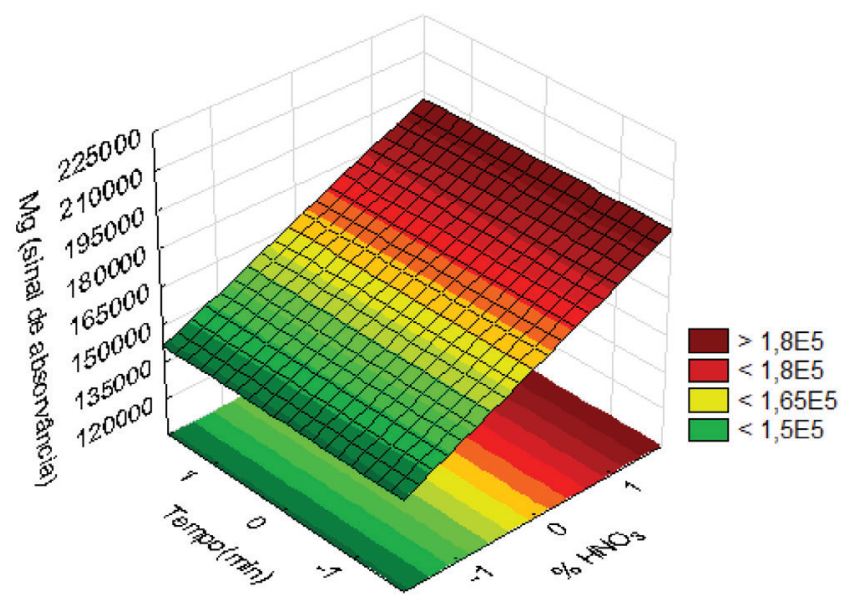

Figura 4. Gráficos de superfície de resposta em função do sinal analítico do $\mathrm{Mg}(\mathrm{tx} \% \mathrm{Ac})$

elementos Fe, Mg, Mo e Na mostrou-se eficiente para a decomposição das amostras de casca de arroz, como comprova os resultados obtidos para os testes de adição e recuperação de analito. Além dos analitos já estudados no DCCR, a condição de preparo de amostras encontrada, para decomposição ácida no sistema sob refluxo, mostrou-se eficiente também para a determinação dos elementos $\mathrm{Al}, \mathrm{Cd}, \mathrm{Cu}, \mathrm{Mn}, \mathrm{Ni}$ e Ti, presentes nas amostras de casca e cinzas de casca de arroz.

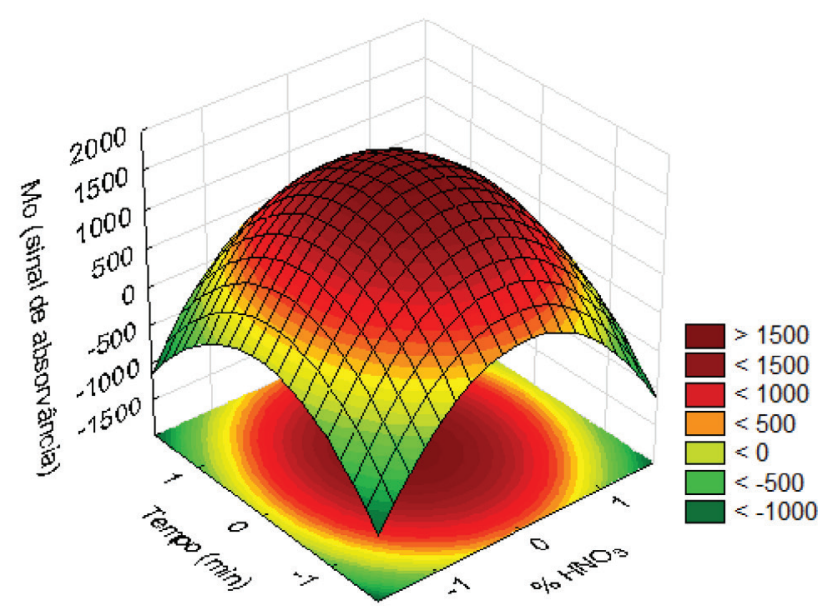

Figura 5. Gráficos de superfície de resposta em função do sinal analítico do $\mathrm{Mo}(\mathrm{tx} \% \mathrm{Ac})$

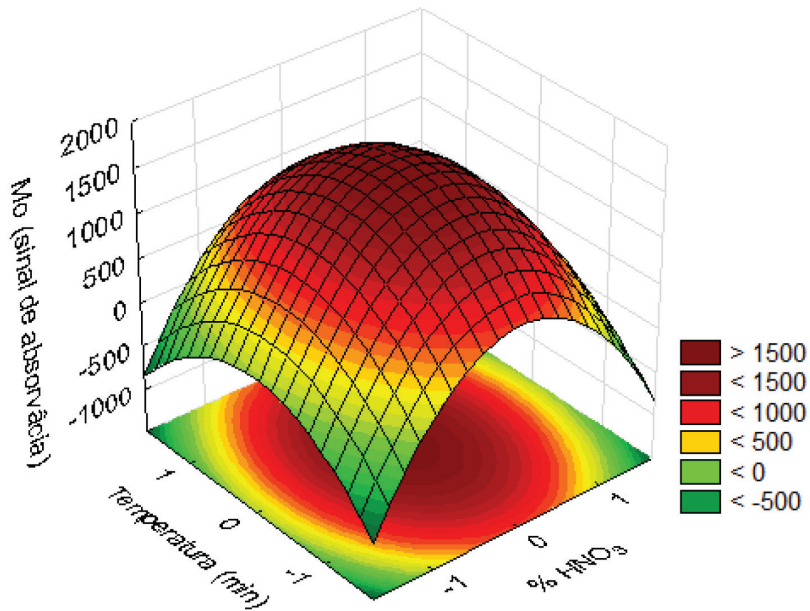

Figura 6. Gráficos de superfície de resposta em função do sinal analítico do $\mathrm{Mo}(\mathrm{Tx} \% \mathrm{Ac})$

O estudo estatístico desenvolvido, mostrou-se uma importante ferramenta para a etapa de preparo de amostras, tendo em vista que possibilitou a obtenção de uma mesma condição para diversos elementos, proporcionou o uso de temperaturas mais baixas, e assegurou qualidade aos resultados obtidos. O uso do DCCR ainda possibilitou realizar um número reduzido de ensaios, comparado aos testes univariados ou a combinação de todas as variáveis estudadas. 


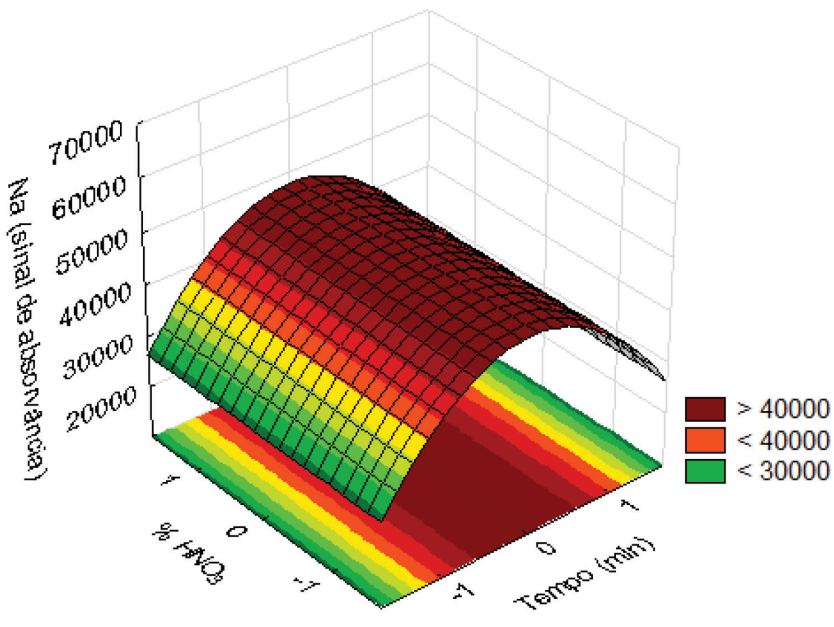

Figura 7. Gráficos de superfície de resposta em função do sinal analítico do $\mathrm{Na}(\% \mathrm{Acxt})$

\section{Parâmetros de mérito}

Os parâmetros de mérito obtidos para a determinação de $\mathrm{Al}, \mathrm{Cd}$, $\mathrm{Cu}, \mathrm{Fe}, \mathrm{Mg}, \mathrm{Mn}, \mathrm{Mo}, \mathrm{Na}, \mathrm{Ni}$, e Ti em amostras de casca de arroz e cinzas de casca de arroz por MIP OES a partir da metodologia proposta estão apresentados na Tabela 5. Como é possível observar, as curvas de calibração para todos os analitos em estudo mostraram bons coeficientes de correlação linear, com $\mathrm{R}>0,999$, bem como apresentaram limites de detecção (LDs) e sensibilidades adequadas para atender ao objetivo que o trabalho se propõe. O limite de detecção do equipamento foi calculado por meio da equação 5 .

$$
\mathrm{LD}=\frac{3 \times \mathrm{S}}{\mathrm{a}}
$$

E o limite de detecção do método foi calculado utilizando também a fórmula 5, porém levando em consideração a massa e o volume utilizados nas análises.

Tabela 5. Parâmetros de Mérito para de $\mathrm{Al}, \mathrm{Cd}, \mathrm{Cu}, \mathrm{Fe}, \mathrm{Mg}, \mathrm{Mn}, \mathrm{Mo}, \mathrm{Na}, \mathrm{Ni}$, e Ti por MIP OES em casca e cinzas de casca de arroz

\begin{tabular}{lccccc}
\hline Analito & $\begin{array}{c}\text { Faixa Linear } \\
\left(\mathrm{mg} \mathrm{L}^{-1}\right)\end{array}$ & $\begin{array}{c}\mathrm{a} \\
\left(\mathrm{L} \mathrm{mg}^{-1}\right)\end{array}$ & $\begin{array}{c}\mathrm{LD} \\
\left(\mathrm{mg} \mathrm{L}^{-1}\right)\end{array}$ & $\begin{array}{c}\mathrm{LD}^{*} \\
\left(\mathrm{mg} \mathrm{kg}^{-1}\right)\end{array}$ & $\mathrm{R}$ \\
\hline $\mathrm{Al}$ & $0,5-5$ & 42700,1 & 0,002 & 0,3986 & 0,99984 \\
$\mathrm{Cd}$ & $0,5-5$ & 37900,7 & 0,0332 & 6,647 & 0,99906 \\
$\mathrm{Cu}$ & $0,5-5$ & 220491 & 0,002 & 0,4051 & 0,99985 \\
$\mathrm{Fe}$ & $0,5-5$ & 14735,5 & 0,0103 & 2,055 & 0,99995 \\
$\mathrm{Mg}$ & $0,5-5$ & 286636 & 0,0011 & 0,2218 & 0,99975 \\
$\mathrm{Mn}$ & $0,5-5$ & 51361,4 & 0,0016 & 0,3171 & 0,99997 \\
$\mathrm{Mo}$ & $0,5-5$ & 44444 & 0,0065 & 1,294 & 0,99996 \\
$\mathrm{Na}$ & $0,5-5$ & 594870 & 0,025 & 4,998 & 0,99964 \\
$\mathrm{Ni}$ & $0,5-5$ & 32078,5 & 0,0608 & 12,17 & 0,99997 \\
$\mathrm{Ti}$ & $0,5-5$ & 53018,7 & 0,0623 & 12,45 & 0,99997 \\
\hline
\end{tabular}

a: coeficiente angular da curva; LD: Limite de detecção do equipamento; LD*: Limite de detecção do método; R: coeficiente da curva de calibração.

\section{Validação da metodologia proposta e aplicação das condições otimizadas}

Com o objetivo de avaliar a exatidão da metodologia proposta, testes de adição e recuperação dos analitos foram realizados utilizando amostras de casca e cinzas de casca de arroz fortificadas e não fortificadas com os analitos de interesse. Nestes testes foram estudados dois pontos de adição de analito, 0,25 e 0,5 ppm. Como citado anteriormente, foi necessária uma diluição de todas as amostras para que a acidez presente não ocasionasse danos ao equipamento, sendo assim, a quantidade adicionada passou a ser 0,5 e 1,0 ppm, para diluição de 2 vezes e 1,25 e 2,5 ppm, para as diluições de 5 vezes. A diluição de 5 vezes se fez necessária, visto que alguns analitos apresentaram resultados de concentração acima da faixa de trabalho estabelecida, necessitando de uma maior diluição das amostras para a análise. Os resultados dos testes de adição e recuperação para as amostras de casca e cinzas da casca de arroz estão descritos nas Tabelas 6 e 7, respectivamente.

De acordo com os resultados apresentados na Tabela 6, pode-se verificar uma faixa de recuperação dos respectivos analitos para as amostras de casca de arroz que variou de 82 a $115 \%$ com valores de desvio padrão relativo (RSDs) inferiores a $10 \%$, o que comprova a exatidão do método proposto, bem como uma precisão adequada, tendo em vista a boa reprodutibilidade dos resultados. Cabe salientar que, o método proposto também se mostrou eficiente para a determinação dos analitos de interesse quando aplicado a amostras de cinzas de casca de arroz (Tabela 7), onde os valores de recuperação dos analitos variaram de 82 a $120 \%$, com RSDs inferiores a $6 \%$.

Assim, podemos dizer que o sistema de decomposição sob refluxo apresentou eficiência na decomposição das amostras de casca e cinzas de casca de arroz possibilitando a subsequente determinação dos analitos. É importante ressaltar que, esta eficiência no processo de preparo das amostras está diretamente relacionada à zona de baixa temperatura do dedo frio, a qual é responsável pela condensação dos vapores gerados, aumentando a capacidade de decomposição.

Através dos resultados apresentados nas Tabelas 6 e 7, também foi possível verificar a aplicabilidade do sistema de decomposição proposto para determinação de elementos voláteis, devido aos bons percentuais de recuperação obtidos para o Cd em ambas as amostras. Por se tratar de um sistema semifechado, certamente, na superfície do dedo frio, é gerada uma película fina de nitratos (decorrente da decomposição com o ácido nítrico), que permite a absorção dos analitos.

De acordo com a normativa vigente da $\mathrm{ABNT}^{13}$, que classifica as cascas de arroz, bem como as cinzas como resíduos e com base nos resultados de concentração obtidos para estas amostras (Tabelas 6 e 7), pôde-se verificar que as concentrações de $\mathrm{Cd}, \mathrm{Cu}$ e $\mathrm{Na}$ ficaram abaixo dos limites máximos permitidos que são respectivamente, $0,005 \mathrm{mg} \mathrm{L}^{-1}, 2,0 \mathrm{mg} \mathrm{L}^{-1}$ e $200 \mathrm{mg} \mathrm{L}^{-1}$. No entanto, as concentrações de Fe tanto para casca de arroz $\left(9,41 \mathrm{mg} \mathrm{L}^{-1}\right)$, quanto para cinzas (3,24 $\left.\mathrm{mg} \mathrm{L}^{-1}\right)$, foram superiores ao limite permitido que é de $0,3 \mathrm{mg}$ $\mathrm{L}^{-1}$. Concentrações acima do permitido também foram obtidas para Mn (casca de arroz (1,28 $\left.\mathrm{mg} \mathrm{L}^{-1}\right) /$ cinzas $\left(5,75 \mathrm{mg} \mathrm{L}^{-1}\right)$ e Al (casca de arroz $\left(0,41 \mathrm{mg} \mathrm{L}^{-1}\right) /$ cinzas $\left(0,60 \mathrm{mg} \mathrm{L}^{-1}\right)$, sendo que os limites para estes elementos são de $0,1 \mathrm{mg} \mathrm{L}^{-1}$ para o $\mathrm{Mn}$ e $0,2 \mathrm{mg} \mathrm{L}^{-1}$ para o $\mathrm{Al}$, o que classifica estas matrizes pela normativa como sendo, resíduos sólidos classe II A - Não inerte.

Os valores de concentração acima do limite estabelecido pela norma corroboram a importância do conhecimento e monitoramento das concentrações de metais presentes nestas matrizes, a fim de evitar possíveis problemas ambientais que possam ser causados quando estas são descartadas como resíduo.

Tendo em vista que os resultados obtidos no teste de adição e recuperação apontaram boa exatidão e precisão, além de baixos limites de detecção do método como mostra a Tabela 5, a mesma condição foi aplicada em todas as amostras de casca e cinza da casca de arroz. Como é possível observar nas Tabelas 8 e 9, respectivamente.

A partir dos resultados apresentados nas Tabelas 8 e 9, foi possível observar pouca variação em relação à concentração dos analitos, 
Tabela 6. Concentrações obtidas em $\mathrm{mg} \mathrm{L}^{-1}$ de $\mathrm{Al}, \mathrm{Cd}, \mathrm{Cu}, \mathrm{Fe}, \mathrm{Mg}, \mathrm{Mn}, \mathrm{Mo}$, $\mathrm{Na}, \mathrm{Ni}$, e Ti em casca de arroz, média da concentração \pm desvio (RSD) e \% de recuperação

\begin{tabular}{|c|c|c|c|}
\hline \multicolumn{4}{|c|}{ Amostras de casca de arroz } \\
\hline Elemento & $\begin{array}{l}\text { Concentração de } \\
\text { analito adicionada }\end{array}$ & $\begin{array}{c}\text { média } \pm \text { desvio } \\
\text { padrão }(\mathrm{RSD})\end{array}$ & $\begin{array}{c}\text { Recuperação } \\
(\%)\end{array}$ \\
\hline & Sem adição & $0,41 \pm 0,04(9,7)$ & \\
\hline \multirow[t]{3}{*}{$\mathrm{Al}$} & Com adição de 0,5 ppm & $0,86 \pm 0,02(2,3)$ & 95 \\
\hline & Com adição de 1,0 ppm & $1,57 \pm 0,07(4,5)$ & 111 \\
\hline & Sem adição & $<\mathrm{LD}$ & \\
\hline \multirow[t]{3}{*}{$\mathrm{Cd}$} & Com adição de 1,25 ppm & $1,30 \pm 0,12(9,2)$ & 104 \\
\hline & Com adição de 2,5 ppm & $2,72 \pm 0,01(0,4)$ & 109 \\
\hline & Sem adição & $<\mathrm{LD}$ & \\
\hline \multirow[t]{3}{*}{$\mathrm{Cu}$} & Com adição de 0,5 ppm & $0,49 \pm 0,01(2,0)$ & 98 \\
\hline & Com adição de 1,0 ppm & $1,11 \pm 0,04(3,6)$ & 111 \\
\hline & Sem adição & $9,41 \pm 0,28(3,0)$ & \\
\hline \multirow[t]{3}{*}{$\mathrm{Fe}$} & Com adição de 1,25 ppm & $12,26 \pm 0,87(7,1)$ & 115 \\
\hline & Com adição de 2,5 ppm & $11,99 \pm 0,69(5,8)$ & 101 \\
\hline & Sem adição & $2,14 \pm 0,09(4,2)$ & \\
\hline \multirow[t]{3}{*}{$\mathrm{Mg}$} & Com adição de 1,25 ppm & $3,54 \pm 0,13(3,7)$ & 104 \\
\hline & Com adição de 2,5 ppm & $4,58 \pm 0,22(4,8)$ & 98 \\
\hline & Sem adição & $1,28 \pm 0,03(2,3)$ & \\
\hline \multirow[t]{3}{*}{$\mathrm{Mn}$} & Com adição de 0,5 ppm & $1,61 \pm 0,13(8,1)$ & 90 \\
\hline & Com adição de 1,0 ppm & $2,22 \pm 0,02(0,9)$ & 97 \\
\hline & Sem adição & $<\mathrm{LD}$ & \\
\hline \multirow[t]{3}{*}{ Mo } & Com adição de 1,25 ppm & $1,42 \pm 0,10(7,0)$ & 114 \\
\hline & Com adição de 2,5 ppm & $2,50 \pm 0,07(2,8)$ & 100 \\
\hline & Sem adição & $0,32 \pm 0,03(9,4)$ & \\
\hline \multirow[t]{3}{*}{$\mathrm{Na}$} & Com adição de 0,5 ppm & $0,67 \pm 0,04(6,0)$ & 82 \\
\hline & Com adição de 1,0 ppm & $1,40 \pm 0,12(8,6)$ & 106 \\
\hline & Sem adição & $1,16 \pm 0,04(3,4)$ & \\
\hline \multirow[t]{3}{*}{$\mathrm{Ni}$} & Com adição de 1,25 ppm & $2,32 \pm 0,03(1,3)$ & 96 \\
\hline & Com adição de 2,5 ppm & $4,07 \pm 0,02(0,5)$ & 110 \\
\hline & Sem adição & $<\mathrm{LD}$ & \\
\hline \multirow[t]{2}{*}{$\mathrm{Ti}$} & Com adição de 0,5 ppm & $0,48 \pm 0,04(8,3)$ & 96 \\
\hline & Com adição de 1,0 ppm & $1,09 \pm 0,07(6,4)$ & 109 \\
\hline
\end{tabular}

tanto para as amostras de casca, como para as amostras de cinzas nos diferentes dias de coleta de cada empresa. No entanto, não houve um padrão de comportamento, quando comparado os resultados de concentração obtidos para os respectivos analitos entre as amostras de casca e cinzas de casca de arroz, já que alguns apresentaram aumento na concentração e outros tiveram suas concentrações consideravelmente diminuídas.

Concentrações maiores em relação às amostras de cinzas, comparadas as amostras de casca, pode ser resultado da incorporação do analito por meio do sistema utilizado durante o processo de queima
Tabela 7. Concentrações obtidas em $\mathrm{mg} \mathrm{L}^{-1}$ de $\mathrm{Al}, \mathrm{Cd}, \mathrm{Cu}, \mathrm{Fe}, \mathrm{Mg}, \mathrm{Mn}$, $\mathrm{Mo}, \mathrm{Na}, \mathrm{Ni}$, e Ti em cinzas de casca de arroz, média \pm desvio (RSD) e \% de recuperação

\begin{tabular}{|c|c|c|c|}
\hline \multicolumn{4}{|c|}{ Amostras de cinzas } \\
\hline Elemento & $\begin{array}{l}\text { Concentração de } \\
\text { analito adicionada }\end{array}$ & $\begin{array}{l}\text { média } \pm \text { desvio } \\
\text { padrão }(\mathrm{RSD})\end{array}$ & $\begin{array}{c}\text { Recuperação } \\
(\%)\end{array}$ \\
\hline & Sem adição & $0,60 \pm 0,01(1,7)$ & \\
\hline \multirow[t]{3}{*}{$\mathrm{Al}$} & Com adição de 1,25 ppm & $1,91 \pm 0,01(0,5)$ & 103 \\
\hline & Com adição de 2,5ppm & $3,71 \pm 0,01(0,3)$ & 120 \\
\hline & Sem adição & $<\mathrm{LD}$ & \\
\hline \multirow[t]{3}{*}{$\mathrm{Cd}$} & Com adição de 1,25 ppm & $1,23 \pm 0,03(2,4)$ & 98 \\
\hline & Com adição de 2,5ppm & $2,51 \pm 0,02(0,8)$ & 100 \\
\hline & Sem adição & $0,21 \pm 0,01(4,8)$ & \\
\hline \multirow[t]{3}{*}{$\mathrm{Cu}$} & Com adição de 1,25 ppm & $1,20 \pm 0,03(2,5)$ & 82 \\
\hline & Com adição de 2,5ppm & $2,49 \pm 0,13(5,2)$ & 92 \\
\hline & Sem adição & $3,24 \pm 0,07(2,2)$ & \\
\hline \multirow[t]{3}{*}{$\mathrm{Fe}$} & Com adição de 1,25 ppm & $4,44 \pm 0,13(3,0)$ & 99 \\
\hline & Com adição de 2,5 ppm & $6,04 \pm 0,19(3,1)$ & 105 \\
\hline & Sem adição & $11,73 \pm 0,03(0,3)$ & \\
\hline \multirow[t]{3}{*}{$\mathrm{Mg}$} & Com adição de 1,25 ppm & $12,83 \pm 0,06(0,5)$ & 99 \\
\hline & Com adição de 2,5 ppm & $14,78 \pm 0,50(3,4)$ & 104 \\
\hline & Sem adição & $5,75 \pm 0,04(0,7)$ & \\
\hline \multirow[t]{3}{*}{ Mn } & Com adição de 1,25 ppm & $7,02 \pm 0,07(1,0)$ & 100 \\
\hline & Com adição de 2,5ppm & $9,18 \pm 0,19(2,1)$ & 111 \\
\hline & Sem adição & $<\mathrm{LD}$ & \\
\hline \multirow[t]{3}{*}{ Mo } & Com adição de 1,25 ppm & $1,02 \pm 0,04(3,9)$ & 82 \\
\hline & Com adição de 2,5ppm & $2,2 \pm 0,01(0,5)$ & 88 \\
\hline & Sem adição & $5,80 \pm 0,06(1,0)$ & \\
\hline \multirow[t]{3}{*}{$\mathrm{Na}$} & Com adição de 1,25 ppm & $7,59 \pm 0,31(4,1)$ & 108 \\
\hline & Com adição de 2,5 ppm & $9,15 \pm 0,33(3,6)$ & 110 \\
\hline & Sem adição & $<\mathrm{LD}$ & \\
\hline \multirow[t]{3}{*}{$\mathrm{Ni}$} & Com adição de 1,25 ppm & $1,13 \pm 0,04(3,5)$ & 90 \\
\hline & Com adição de 2,5ppm & $2,26 \pm 0,11(4,9)$ & 90 \\
\hline & Sem adição & $<\mathrm{LD}$ & \\
\hline \multirow[t]{2}{*}{$\mathrm{Ti}$} & Com adição de 1,25 ppm & $1,20 \pm 0,06(5,0)$ & 96 \\
\hline & Com adição de 2,5ppm & $2,41 \pm 0,07(2,9)$ & 96 \\
\hline
\end{tabular}

da casca de arroz ou no período de estocagem das cinzas resultantes. Concentrações menores nas amostras de cinzas estão diretamente relacionadas com as perdas de elementos por volatilização, tendo em vista que, durante o processo de queima da casca de arroz, temperaturas superiores a $500{ }^{\circ} \mathrm{C}$ são aplicadas e assim perdas significativas de alguns elementos durante este processo são justificadas. ${ }^{8}$

Com base nos resultados apresentados nas respectivas tabelas, verifica-se que o resíduo final do processo de queima da casca de arroz apresenta concentrações consideráveis de $\mathrm{Fe}, \mathrm{Mg}, \mathrm{Mn}$, Ti e $\mathrm{Na}$ o que vem a contribuir com a possível aplicação deste resíduo na 
Tabela 8. Concentrações obtidas em $\mathrm{mg} \mathrm{Kg}^{-1}$ de Al, $\mathrm{Cd}, \mathrm{Cu}, \mathrm{Fe}, \mathrm{Mg}, \mathrm{Mn}, \mathrm{Mo}, \mathrm{Na}, \mathrm{Ni}$, e Ti em amostras de casca de arroz, média \pm desvio (RSD)

\begin{tabular}{|c|c|c|c|c|c|}
\hline \multirow{2}{*}{ Amostras } & \multicolumn{5}{|c|}{ Elementos } \\
\hline & $\mathrm{Al}$ & $\mathrm{Cd}$ & $\mathrm{Cu}$ & $\mathrm{Fe}$ & $\mathrm{Mg}$ \\
\hline \multicolumn{6}{|c|}{ Empresa 1} \\
\hline $1^{\circ} \mathrm{dia}$ & $70,48 \pm 4,97(7,05)$ & $<\mathrm{LD}$ & $20,33 \pm 1,66(8,14)$ & $1298 \pm 87(6,77)$ & $322,7 \pm 16,6(5,17)$ \\
\hline $2^{\circ} \mathrm{dia}$ & $43,64 \pm 0,47(1,07)$ & $<\mathrm{LD}$ & $6,477 \pm 0,172(2,66)$ & $1197 \pm 26(2,23)$ & $391,3 \pm 3,1(0,79)$ \\
\hline $3^{\circ} \mathrm{dia}$ & $79,68 \pm 3,87(4,85)$ & $<\mathrm{LD}$ & $13,75 \pm 0,53(3,87)$ & $1252 \pm 41(3,28)$ & $364,4 \pm 11,1(3,05)$ \\
\hline $4^{\circ} \mathrm{dia}$ & $55,49 \pm 2,13(3,84)$ & $<\mathrm{LD}$ & $8,580 \pm 0,450(5,27)$ & $1209 \pm 43(3,59)$ & $375,8 \pm 30,1(8,01)$ \\
\hline $5^{\circ}$ dia & $65,42 \pm 3,76(5,75)$ & $<\mathrm{LD}$ & $43,06 \pm 0,72(1,68)$ & $1563 \pm 40(2,57)$ & $345,5 \pm 19,0(5,49)$ \\
\hline \multicolumn{6}{|c|}{ Empresa 2} \\
\hline $1^{\circ} \mathrm{dia}$ & $45,48 \pm 2,50(5,49)$ & $<\mathrm{LD}$ & $27,45 \pm 1,22(4,46)$ & $1144,52 \pm 76,76(6,71)$ & $294,0 \pm 15,3(5,20)$ \\
\hline $2^{\circ} \mathrm{dia}$ & $46,63 \pm 0,49(1,05)$ & $<\mathrm{LD}$ & $<\mathrm{LD}$ & $516,8 \pm 16,0(3,10)$ & $435,9 \pm 15,0(3,45)$ \\
\hline $3^{\circ} \mathrm{dia}$ & $42,73 \pm 0,55(1,29)$ & $<\mathrm{LD}$ & $<\mathrm{LD}$ & $430,6 \pm 0,1(0,20)$ & $450,8 \pm 5,6(1,24)$ \\
\hline $4^{\circ} \mathrm{dia}$ & $59,35 \pm 4,63(7,79)$ & $<\mathrm{LD}$ & $<\mathrm{LD}$ & $538,1 \pm 3,2(0,59)$ & $418,5 \pm 6,4(1,52)$ \\
\hline $5^{\circ} \mathrm{dia}$ & $91,76 \pm 4,09(4,46)$ & $<\mathrm{LD}$ & $<\mathrm{LD}$ & $413,6 \pm 3,9(0,93)$ & $410,6 \pm 9,5(2,31)$ \\
\hline \multicolumn{6}{|c|}{ Empresa 3} \\
\hline $1^{\circ} \mathrm{dia}$ & $51,46 \pm 1,42(2,75)$ & $<\mathrm{LD}$ & $<\mathrm{LD}$ & $429,4 \pm 26,8(6,23)$ & $579,5 \pm 17,7(3,05)$ \\
\hline $2^{\circ} \mathrm{dia}$ & $167,4 \pm 4,5(2,72)$ & $<\mathrm{LD}$ & $<\mathrm{LD}$ & $746,9 \pm 0,3(0,04)$ & $500,5 \pm 8,1(1,61)$ \\
\hline $3^{\circ} \mathrm{dia}$ & $45,09 \pm 5,44(12,48)$ & $<\mathrm{LD}$ & $<\mathrm{LD}$ & $524,5 \pm 34,9(6,66)$ & $623,7 \pm 40,7(6,53)$ \\
\hline $4^{\circ} \mathrm{dia}$ & $58,00 \pm 2,00(3,45)$ & $<\mathrm{LD}$ & $<\mathrm{LD}$ & $577,6 \pm 13,6(2,36)$ & $557,8 \pm 19,2(3,44)$ \\
\hline$\underline{5^{\circ} \mathrm{dia}}$ & $71,40 \pm 4,59(6,43)$ & $<\mathrm{LD}$ & $<\mathrm{LD}$ & $519,9 \pm 15,5(2,98)$ & $594,0 \pm 15,4(2,59)$ \\
\hline \multirow{2}{*}{ Amostras } & \multicolumn{5}{|c|}{ Elementos } \\
\hline & $\mathrm{Mn}$ & Mo & $\mathrm{Na}$ & $\mathrm{Ni}$ & $\mathrm{Ti}$ \\
\hline \multicolumn{6}{|c|}{ Empresa 1} \\
\hline $1^{\circ} \mathrm{dia}$ & $234,9 \pm 15,6(6,63)$ & $<\mathrm{LD}$ & $131,1 \pm 5,6(4,29)$ & $146,3 \pm 7,5(5,14)$ & $<\mathrm{LD}$ \\
\hline $2^{\circ} \mathrm{dia}$ & $239,7 \pm 1,4(0,57)$ & $<\mathrm{LD}$ & $138,9 \pm 5,7(4,10)$ & $136,3 \pm 3,6(2,66)$ & $<\mathrm{LD}$ \\
\hline $3^{\circ} \mathrm{dia}$ & $232,6 \pm 2,9(1,23)$ & $<\mathrm{LD}$ & $129,3 \pm 2,5(1,94)$ & $128,6 \pm 2,8(2,14)$ & $<\mathrm{LD}$ \\
\hline $4^{\circ} \mathrm{dia}$ & $243,4 \pm 9,9(4,05)$ & $<\mathrm{LD}$ & $141,1 \pm 4,4(3,09)$ & $139,1 \pm 4,6(3,31)$ & $<\mathrm{LD}$ \\
\hline $5^{\circ}$ dia & $252,8 \pm 13,3(5,24)$ & $<\mathrm{LD}$ & $135,9 \pm 6,2(4,60)$ & $182,4 \pm 0,5(0,29)$ & $<\mathrm{LD}$ \\
\hline \multicolumn{6}{|c|}{ Empresa 2} \\
\hline $1^{\circ} \mathrm{dia}$ & $160,4 \pm 7,0(4,36)$ & $<\mathrm{LD}$ & $74,26 \pm 0,01(0,02)$ & $<\mathrm{LD}$ & $16,93 \pm 0,78(4,59)$ \\
\hline $2^{\circ} \mathrm{dia}$ & $177,2 \pm 7,2(4,09)$ & $<\mathrm{LD}$ & $70,70 \pm 4,79(6,78)$ & $<\mathrm{LD}$ & $21,89 \pm 1,21(5,52)$ \\
\hline $3^{\circ} \mathrm{dia}$ & $157,1 \pm 8,3(5,31)$ & $<\mathrm{LD}$ & $81,04 \pm 5,74(7,09)$ & $<\mathrm{LD}$ & $21,93 \pm 0,16(0,74)$ \\
\hline $4^{\circ} \mathrm{dia}$ & $187,6 \pm 3,2(1,73)$ & $<\mathrm{LD}$ & $90,55 \pm 5,20(5,74)$ & $<\mathrm{LD}$ & $23,22 \pm 1,12(4,84)$ \\
\hline $5^{\circ}$ dia & $199,2 \pm 14,3(7,18)$ & $<\mathrm{LD}$ & $83,41 \pm 5,35(6,42)$ & $<\mathrm{LD}$ & $27,09 \pm 0,91(3,34)$ \\
\hline \multicolumn{6}{|c|}{ Empresa 3} \\
\hline $1^{\circ} \mathrm{dia}$ & $238,5 \pm 8,3(3,48)$ & $<\mathrm{LD}$ & $149,0 \pm 4,2(2,79)$ & $<\mathrm{LD}$ & $47,17 \pm 4,38(9,29)$ \\
\hline $2^{\circ} \mathrm{dia}$ & $253,1 \pm 6,5(2,56)$ & $<\mathrm{LD}$ & $144,5 \pm 1,9(1,33)$ & $<\mathrm{LD}$ & $45,21 \pm 2,00(4,43)$ \\
\hline $3^{\circ} \mathrm{dia}$ & $260,9 \pm 18,5(7,10)$ & $<\mathrm{LD}$ & $146,9 \pm 4,1(2,79)$ & $<\mathrm{LD}$ & $47,65 \pm 4,19(8,80)$ \\
\hline $4^{\circ} \mathrm{dia}$ & $226,2 \pm 21,7(9,58)$ & $<\mathrm{LD}$ & $133,9 \pm 6,5(4,89)$ & $<\mathrm{LD}$ & $123,4 \pm 1,7(1,33)$ \\
\hline $5^{\circ} \mathrm{dia}$ & $241,3 \pm 13,8(5,72)$ & $<\mathrm{LD}$ & $148,3 \pm 8,2(5,54)$ & $<\mathrm{LD}$ & $67,20 \pm 1,48(2,20)$ \\
\hline
\end{tabular}

agricultura como adubo do solo, tendo em vista que estes nutrientes são essenciais para o bom desenvolvimento dos seres vivos. ${ }^{14,15}$

Cabe salientar que, a caracterização elementar destas amostras, é de grande importância, o que vem a colaborar de forma significativa no destino destes resíduos. De acordo com a normativa vigente da $\mathrm{ABNT}^{13}$, as amostras de casca e cinza de casca de arroz apresentadas nas Tabelas 8 e 9, são classificadas como resíduos sólidos classe II A - Não inerte, pois apresentam as concentrações (quando transformadas para $\mathrm{mg} / \mathrm{L}$ ) de alguns elementos acima do limite permitido na normativa.

\section{CONCLUSÃO}

Através dos resultados obtidos foi possível observar que o método proposto de decomposição ácida com sistema de refluxo utilizado para o preparo das amostras de casca e cinzas de casca de arroz apresentou-se como um método eficiente, seguro e simples, apresentando exatidão e precisão adequadas para a análise de $\mathrm{Al}$, $\mathrm{Cd}, \mathrm{Cu}, \mathrm{Fe}, \mathrm{Mg}, \mathrm{Mn}, \mathrm{Mo}, \mathrm{Na}, \mathrm{Ni}$, e Ti por MIP OES. Sendo assim, uma alternativa promissora aos métodos convencionais de análise. Os valores de recuperação encontrados para ambas as amostras confirmam a exatidão do método. A utilização do planejamento estatístico durante o processo de decomposição das amostras possibilitou, com um número menor de ensaios, uma avaliação mais eficiente em relação aos fatores estudados, auxiliando na otimização das condições de trabalho. Com base no cenário atual, aonde a casca de arroz vem sendo amplamente empregada como fonte de energia, gerando as cinzas como subproduto, a caracterização elementar dessas matrizes vem auxiliar as empresas em relação ao destino deste resíduo, bem como ao aproveitamento adequado de matéria-prima, o que irá resultar em benefícios em relação ao processo de conservação ambiental. 
Tabela 9. Concentrações obtidas em mg Kg ${ }^{-1}$ de Al, $\mathrm{Cd}, \mathrm{Cu}, \mathrm{Fe}, \mathrm{Mg}, \mathrm{Mn}, \mathrm{Mo}, \mathrm{Na}, \mathrm{Ni}$, e Ti em amostras de cinzas de casca de arroz , média \pm desvio (RSD)

\begin{tabular}{|c|c|c|c|c|c|}
\hline \multirow{2}{*}{ Amostras } & \multicolumn{5}{|c|}{ Elementos } \\
\hline & $\mathrm{Al}$ & $\mathrm{Cd}$ & $\mathrm{Cu}$ & $\mathrm{Fe}$ & $\mathrm{Mg}$ \\
\hline \multicolumn{6}{|c|}{ Empresa 1} \\
\hline $1^{\circ} \mathrm{dia}$ & $677,9 \pm 35,1(5,18)$ & $<\mathrm{LD}$ & $33,65 \pm 0,46(1,37)$ & $614,8 \pm 5,6(0,90)$ & $1098 \pm 12(1,06)$ \\
\hline $2^{\circ}$ dia & $105,0 \pm 5,2(4,91)$ & $<\mathrm{LD}$ & $33,25 \pm 0,12(0,37)$ & $462,9 \pm 5,6(1,20)$ & $1083 \pm 12(1,09)$ \\
\hline $3^{\circ} \mathrm{dia}$ & $102,9 \pm 6,7(6,48)$ & $<\mathrm{LD}$ & $33,48 \pm 0,14(0,40)$ & $451,0 \pm 10,7(2,37)$ & $1066 \pm 6(0,58)$ \\
\hline $4^{\circ} \mathrm{dia}$ & $105,6 \pm 7,9(7,47)$ & $<\mathrm{LD}$ & $33,42 \pm 0,38(1,13)$ & $477,8 \pm 21,5(4,50)$ & $1001 \pm 5(0,50)$ \\
\hline $5^{\circ} \mathrm{dia}$ & $105,8 \pm 10,4(9,79)$ & $<\mathrm{LD}$ & $33,62 \pm 0,18(0,54)$ & $418,3 \pm 11,9(2,83)$ & $988,5 \pm 5,6(0,56)$ \\
\hline \multicolumn{6}{|c|}{ Empresa 2} \\
\hline $1^{\circ}$ dia & $201,5 \pm 4,0(1,99)$ & $<\mathrm{LD}$ & $92,01 \pm 0,38(0,41)$ & $927,9 \pm 28,6(3,09)$ & $2657 \pm 34(1,30)$ \\
\hline $2^{\circ} \mathrm{dia}$ & $205,6 \pm 13,2(6,42)$ & $<\mathrm{LD}$ & $91,21 \pm 0,76(0,84)$ & $919,2 \pm 37,4(4,07)$ & $2605 \pm 17(0,64)$ \\
\hline $3^{\circ} \mathrm{dia}$ & $184,4 \pm 2,6(1,42)$ & $<\mathrm{LD}$ & $81,26 \pm 0,52(0,64)$ & $864,9 \pm 13,0(1,50)$ & $2696 \pm 15(0,56)$ \\
\hline $4^{\circ} \mathrm{dia}$ & $212,5 \pm 20,9(9,82)$ & $<\mathrm{LD}$ & $93,32 \pm 1,51(1,61)$ & $906,5 \pm 9,2(1,01)$ & $2639 \pm 20(0,77)$ \\
\hline $5^{\circ} \mathrm{dia}$ & $177,7 \pm 4,3(2,43)$ & $<\mathrm{LD}$ & $81,91 \pm 1,07(1,31)$ & $843,3 \pm 11,9(1,41)$ & $2670 \pm 30(1,14)$ \\
\hline \multicolumn{6}{|c|}{ Empresa 3} \\
\hline $1^{\circ}$ dia & $74,14 \pm 4,25(5,73)$ & $<\mathrm{LD}$ & $36,49 \pm 0,54(1,47)$ & $332,1 \pm 8,7(2,61)$ & $674,5 \pm 30,2(4,48)$ \\
\hline $2^{\circ} \mathrm{dia}$ & $76,78 \pm 1,80(2,34)$ & $<\mathrm{LD}$ & $36,07 \pm 0,19(0,53)$ & $359,4 \pm 16,9(4,70)$ & $618,7 \pm 1,1(0,17)$ \\
\hline $3^{\circ} \mathrm{dia}$ & $70,95 \pm 3,64(5,13)$ & $<\mathrm{LD}$ & $36,26 \pm 0,25(0,70)$ & $355,6 \pm 11,8(3,31)$ & $655,5 \pm 23,2(3,54)$ \\
\hline $4^{\circ}$ dia & $72,82 \pm 2,44(3,35)$ & $<\mathrm{LD}$ & $36,16 \pm 0,14(0,40)$ & $308,8 \pm 5,8(1,87)$ & $617,7 \pm 17,6(2,85)$ \\
\hline $5^{\circ} \mathrm{dia}$ & $72,38 \pm 0,94(1,30)$ & $<\mathrm{LD}$ & $36,43 \pm 0,24(0,67)$ & $294,9 \pm 5,7(1,95)$ & $626,7 \pm 11,5(1,83)$ \\
\hline \multirow{2}{*}{ Amostras } & \multicolumn{5}{|c|}{ Elementos } \\
\hline & $\mathrm{Mn}$ & Mo & $\mathrm{Na}$ & $\mathrm{Ni}$ & $\mathrm{Ti}$ \\
\hline \multicolumn{6}{|c|}{ Empresa 1} \\
\hline $1^{\circ} \mathrm{dia}$ & $597,0 \pm 10,4(1,75)$ & $<\mathrm{LD}$ & $382,2 \pm 19,7(5,15)$ & $360,7 \pm 3,1(0,86)$ & $605,2 \pm 22,6(3,73)$ \\
\hline $2^{\circ} \mathrm{dia}$ & $581,6 \pm 5,8(0,99)$ & $<\mathrm{LD}$ & $371,7 \pm 13,2(3,55)$ & $353,9 \pm 2,7(0,76)$ & $580,4 \pm 13,3(2,30)$ \\
\hline $3^{\circ}$ dia & $568,3 \pm 3,8(0,67)$ & $<\mathrm{LD}$ & $345,4 \pm 6,5(1,88)$ & $348,6 \pm 3,9(1,13)$ & $554,6 \pm 5,0(0,91)$ \\
\hline $4^{\circ} \mathrm{dia}$ & $537,1 \pm 3,3(0,62)$ & $<\mathrm{LD}$ & $330,5 \pm 1,3(0,40)$ & $350,4 \pm 5,0(1,43)$ & $541,4 \pm 3,0(0,56)$ \\
\hline $5^{\circ}$ dia & $514,6 \pm 2,5(0,48)$ & $<\mathrm{LD}$ & $320,6 \pm 16,3(5,10)$ & $340,9 \pm 3,1(0,90)$ & $534,1 \pm 2,1(0,40)$ \\
\hline \multicolumn{6}{|c|}{ Empresa 2} \\
\hline $1^{\circ}$ dia & $1536 \pm 29(1,86)$ & $168,0 \pm 0,6(0,35)$ & $1675 \pm 10(0,62)$ & $<\mathrm{LD}$ & $502,4 \pm 2,0(0,41)$ \\
\hline $2^{\circ}$ dia & $1533 \pm 11(0,70)$ & $170,7 \pm 0,8(0,48)$ & $1674 \pm 20(1,18)$ & $<\mathrm{LD}$ & $497,5 \pm 4,4(0,88)$ \\
\hline $3^{\circ} \mathrm{dia}$ & $1196 \pm 16(1,30)$ & $170,7 \pm 1,3(0,77)$ & $1511 \pm 26(1,72)$ & $<\mathrm{LD}$ & $495,0 \pm 1,0(0,21)$ \\
\hline $4^{\circ} \mathrm{dia}$ & $1572 \pm 37(2,34)$ & $172,9 \pm 0,9(0,54)$ & $1693 \pm 36(2,10)$ & $<\mathrm{LD}$ & $499,6 \pm 15,6(3,12)$ \\
\hline $5^{\circ}$ dia & $1218 \pm 24(1,95)$ & $175,0 \pm 0,6(0,31)$ & $1501 \pm 25(1,64)$ & $<\mathrm{LD}$ & $483,6 \pm 2,8(0,57)$ \\
\hline \multicolumn{6}{|c|}{ Empresa 3} \\
\hline $1^{\circ} \mathrm{dia}$ & $285,4 \pm 9,5(3,32)$ & $<\mathrm{LD}$ & $280,1 \pm 14,4(5,14)$ & $<\mathrm{LD}$ & $500,2 \pm 4,8(0,97)$ \\
\hline $2^{\circ} \mathrm{dia}$ & $262,5 \pm 2,0(0,74)$ & $<\mathrm{LD}$ & $267,3 \pm 9,9(3,71)$ & $<\mathrm{LD}$ & $489,7 \pm 3,3(0,68)$ \\
\hline $3^{\circ} \mathrm{dia}$ & $271,7 \pm 11,0(4,05)$ & $<\mathrm{LD}$ & $275,0 \pm 7,0(2,54)$ & $<\mathrm{LD}$ & $484,3 \pm 1,8(0,37)$ \\
\hline $4^{\circ} \mathrm{dia}$ & $261,0 \pm 1,8(0,69)$ & $<\mathrm{LD}$ & $260,1 \pm 10,8(4,16)$ & $<\mathrm{LD}$ & $477,9 \pm 3,5(0,73)$ \\
\hline $5^{\circ} \mathrm{dia}$ & $260,0 \pm 6,9(2,64)$ & $<\mathrm{LD}$ & $258,0 \pm 8,7(3,37)$ & $<\mathrm{LD}$ & $470,3 \pm 4,5(0,95)$ \\
\hline
\end{tabular}

\section{REFERÊNCIAS}

1. http://www.agricultura.gov.br/vegetal/culturas/arroz/saiba-mais, acessada em Novembro 2016.

2. Walter, M.; Marchezan, E.; Avila, L. A. D.; Ciência Rural 2008, 38, 1184.

3. Folletto, E. L.; Hoffmann, R.; Hoffmann, R. S.; Portugal Jr, U. L.; Jahn, S. L.; Quim Nova 2005, 28, 1055.

4. http://www.habitare.org.br/pdf/publicacoes/arquivos/132.pdf, acessada em Dezembro 2016

5. Zucco, L. L.; Beraldo, A. L.; Engenharia Agrícola 2008, 28, 217.

6. Ferreira, C. S.; Dissertação de mestrado, Universidade Federal de Santa Catarina, Brasil, 2005.

7. Fernandes, A.; Tese de Doutorado, Universidade de São Paulo, Brasil, 2006.

8. Krug, F. J.; Métodos de Preparo de Amostras: Fundamentos sobrepreparo de amostras orgânicas e inorgânicas para análise elementar, $1^{\text {a }}$ ed., Piracicaba: Edição do autor, São Paulo, 2010.

9. Oreste, E. Q.; Jesus, A.; Oliveira, R. M.; Silva, M. M.; Vieira, M. A.; Ribeiro, A. S.; Microchem. J. 2013, 109, 5.

10. Oreste, E. Q.; Souza, A. O.; Pereira, C. C.; Lisboa, M. T.; Cidade, M. J. A.; Vieira, M. A.; Cadore. S.; Ribeiro, A. S.; Food Anal. Methods 2015, 9, 777.

11. Oliveira, R. de M.; Antunes, A. C. N.; Vieira, M. A.; Medina, A. L.; Ribeiro, A. S.; Microchem. J. 2016, 124, 402.

12. Barros Neto, B.; Scarminio, I. S.; Bruns, R. E., Planejamento e Otimização de Experimentos, $2^{\circ}$ ed., Ed. Unicamp: Campinas, 1995.

13. ABNT (Associação Brasileira de Normas Técnicas) - NBR 10004 Resíduos Sólidos: Classificação; Rio de Janeiro, 2004.

14. Darolt, M. R.; Osaki F.; Resumos do IX Congresso de iniciação Científica em Ciências Agrárias, Curitiba, Brasil, 1989.

15. Rigau, A.; Los Abonos. Su Preparación y Empleo. Guia para o Agricultor Prático, $2^{\text {th }}$.ed., Editorial Sintes: Barcelona, 1978. 